\title{
MONITORAMENTO DA DESESTABILIZAÇÃO TÉRMICA DE EMULSÕES DE UM FLUIDO DE CORTE VIA ESPECTROSCOPIA UV-VIS
}

\author{
V. POSTAL ${ }^{1 *}$, F. A. D. DA ROCHA ${ }^{1}$, R. GUARDANI ${ }^{1}$ \\ ${ }^{1}$ Escola Politécnica da Universidade de São Paulo, Departamento de Engenharia Química \\ *e-mail: victor.postal@usp.br
}

\begin{abstract}
RESUMO
A utilização de fluidos de corte na indústria metal-mecânica é de relevante importância para a eficiência do sistema produtivo como um todo, possuindo ação lubrificante e refrigerante e prevenindo o desgaste de peças e ferramentas. Nos processos de usinagem, emulsões desses fluidos estão sujeitas a ciclos de aquecimento consequentes do calor gerado na área de corte, o que promove a aceleração de reações químicas e a intensificação do movimento Browniano. Como principal consequência, destaca-se o aumento da taxa de coalescência de gotas, o que induz a desestabilização destas emulsões. Neste contexto, investigou-se a estabilidade térmica de emulsões de um fluido de corte comercial sujeitas a ciclos de aquecimento programados, empregando-se a espectroscopia UV-Vis como ferramenta de monitoramento. Os ciclos propostos visaram analisar o impacto de fatores como concentração de fase dispersa, perda de meio contínuo por evaporação, diluição e tempo de aquecimento. Por meio do cálculo do wavelength exponent das emulsões, verificou-se a diminuição deste expoente ao longo dos ciclos em todos os casos estudados, o que foi acompanhado pelo aumento progressivo do tamanho médio de gota. Constatou-se também que o intervalo de concentração de fase dispersa analisada não impacta na estabilidade das emulsões, assim como a reposição de fase contínua evaporada a cada ciclo, ressaltando-se o tempo de aquecimento como fator principal de desestabilização.
\end{abstract}

\section{INTRODUÇÃO}

Um dos materiais de maior importância nos processos de usinagem é o denominado fluido de corte, o qual pode ser definido como sendo líquidos ou gases aplicados na ferramenta e no material que está sendo usinado a fim de facilitar a operação de corte (BAUMEISTER et al., 1978). Neste contexto, o emprego do fluido de corte aumenta a vida útil da ferramenta, minimiza a geração de calor durante o processo e auxilia na remoção dos cavacos, melhorando, assim, a eficiência do sistema produtivo (OLIVEIRA; ALVES, 2007). Por sua vez, do ponto de vista econômico e ambiental, é de grande importância o conhecimento do ciclo de vida desse tipo de material, visando-se sua disposição final no momento e no local corretos, minimizando-se, assim, seu descarte.

Dependendo da operação e do material da peça a ser usinada, emulsões de fluidos de corte podem conter uma concentração volumétrica de fase dispersa entre 1 e $20 \%$ em água, sendo $5 \%$ o valor de diluição mais empregado (BYERS, 2006), atingindo tamanhos de gota inferiores a $0,1 \mu \mathrm{m}$ em alguns casos (DELUHERY; RAJAGOPALAN, 2005).

$\mathrm{Na}$ indústria metal-mecânica, as emulsões de fluido de corte estão sujeitas a ciclos de aquecimento, estando submetidas, 
assim, a elevadas temperaturas, o que causa a aceleração de sua desestabilização por meio da diminuição da viscosidade e do aumento da taxa de coalescência de gotas (GLASSE et al., 2014). Devido ao fato de as propriedades das emulsões, como reologia e estabilidade, serem fortemente determinadas por seu tamanho de gota (HADNADEV et al., 2013), a compreensão do impacto de ciclos de aquecimento no crescimento do tamanho médio de gota de emulsões de fluidos de corte torna-se de fundamental importância para a indústria, o que tem intensificado a utilização de técnicas espectroscópicas para o monitoramento in-line de tais emulsões, permitindo-se a obtenção de dados relacionados a processos de desestabilização em tempo real (ASSENHAIMER et al., 2013).

Para sistemas monodispersos possuindo partículas não absorventes de luz, define-se o wavelength exponent ( $z$ ), também conhecido como expoente de Ångström, pela Equação 1 (REDDY; FOGLER, 1981):

$z=\frac{d \ln (\tau)}{d \ln (1 / \lambda)}$

Segundo Deluhery e Rajagopalan (2005), uma rápida diminuição do valor deste coeficiente é um indicativo do crescimento das gotas da fase dispersa, enquanto que valores aproximadamente constantes ou pequenas variações demonstram que a emulsão é estável. Esta técnica tem se demonstrado promissora, podendo ser utilizada como um critério quantitativo no monitoramento da desestabilização de emulsões de fluidos de corte (GLASSE et al., 2013).

\section{MATERIAIS E MÉTODOS}

Amostras de um fluido de corte comercial foram utilizadas no preparo de emulsões com concentração mássica de $5 \%$ de fase dispersa, sendo a fase contínua composta por água destilada. As principais características do fluido de corte utilizado estão resumidas na Tabela 1 .

Tabela 1 - Propriedades do fluido de corte utilizado no estudo.

\begin{tabular}{cc}
\hline Propriedade & Valor \\
\hline Viscosidade cinemática a & 73 \\
$20{ }^{\circ} \mathrm{C}\left(\mathrm{mm}^{2} / \mathrm{s}\right)$ & 960 \\
Densidade a $20{ }^{\circ} \mathrm{C}\left(\mathrm{kg} / \mathrm{m}^{3}\right)$ & 9,24 \\
$\mathrm{pH}(5 \%)$ & Marrom \\
Cor & 40 \\
Conteúdo de óleo $(\%)$ & 1,27 \\
Índice de refração &
\end{tabular}

Fonte: Jokisch GmbH (2015).

As emulsões foram preparadas em erlenmeyers de vidro borossilicato e respeitou-se uma rotina selecionada de modo a tornar os ensaios reprodutíveis, a qual era composta pelas seguintes etapas:

1. Pesagem da água destilada em uma balança analítica modelo Adventurer (Ohaus);

2. Tara da balança e adição do fluido de corte sobre a superfície da água até se completar a massa total de emulsão;

3. Agitação manual imediata após a adição do fluido de corte.

Verificou-se que a agitação manual era suficiente para atingir o menor tamanho médio de gota possível, não havendo a necessidade de se utilizar outro método para a dispersão do fluido a fim de se garantir a reprodutibilidade dos ensaios.

Ao longo dos experimentos, a massa das emulsões era monitorada em uma balança analítica modelo AS 2000C (Marte) e a aquisição de dados relacionados à temperatura se dava por meio de um termopar TH-096 (Instrutherm). A amostra submetida aos ciclos de aquecimento era aquecida em um aquecedor magnético modelo 752A (Fisatom) 
à $300{ }^{\circ} \mathrm{C}$ e resfriada em um reservatório contendo água em equilíbrio térmico com um banho ultratermostático NT 281 (Novatecnica) à $7{ }^{\circ} \mathrm{C}$, como pode ser visto na Figura 1.

Figura 1 - Aparato experimental utilizado nos ensaios. (a) chapa aquecedora; (b) erlenmeyer usado para o aquecimento da emulsão;

(c) condensador; (d) reservatório de água fria em equilíbrio térmico com um banho

ultratermostático

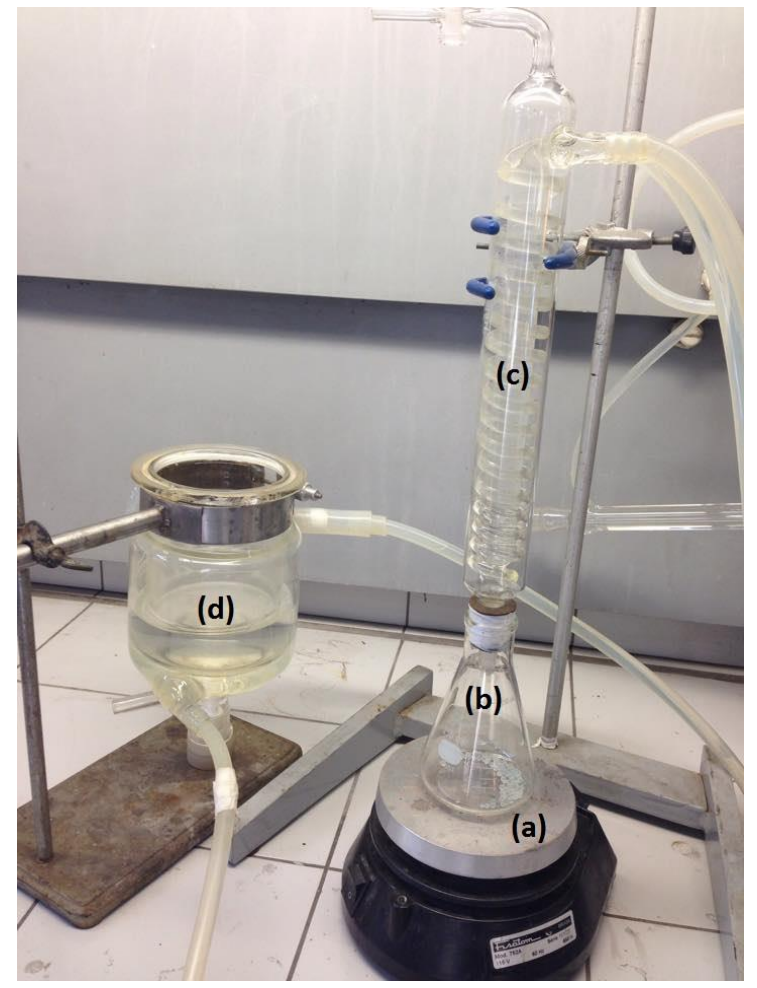

Finalmente, os tamanhos médios de gota eram aferidos em triplicata por meio da técnica de espectroscopia por correlação de fótons em um N4 Plus Submicron Particle Analyzer (Beckman-Coulter) e os espectros de extinção de luz eram obtidos através de um espectrômetro HR 2000+ES (Ocean Optics) com uma fonte de luz DH 2000-BAL (200$1100 \mathrm{~nm}$ ), possuindo uma ponteira com diâmetro $6,35 \mathrm{~mm}$, comprimento de $127 \mathrm{~mm}$ e caminho óptico de $2 \mathrm{~mm}$.
2.1 Levantamento das Curvas de Aquecimento e Resfriamento de uma Emulsão de Fluido de Corte

Em um erlenmeyer de vidro borossilicato, aqueceu-se $50 \mathrm{~g}$ de uma emulsão contendo $5 \%$ em massa do fluido de corte mencionado na Tabela 1 em uma chapa metálica aquecida a $300{ }^{\circ} \mathrm{C}$. Com o auxílio de um termopar, aferiu-se a temperatura da emulsão a cada $15 \mathrm{~s}$ até esta atingir sua temperatura de ebulição. Em seguida, a mesma foi transferida para um reservatório contendo água em equilíbrio térmico com um banho ultratermostático à $7{ }^{\circ} \mathrm{C}$, medindo-se sua temperatura a cada $15 \mathrm{~s}$ até o sistema atingir a temperatura ambiente (admitido como sendo em torno de $25^{\circ} \mathrm{C}$ ).

\subsection{Monitoramento da Desestabilização de Emulsões Submetidas a Ciclos de Aquecimento em Sistema Aberto}

Preparou-se $50 \mathrm{~g}$ de uma emulsão contendo $5 \%$ em massa do fluido de corte descrito anteriormente respeitando-se a rotina de preparo mencionada. $\mathrm{O}$ aquecimento se deu elevando-se a temperatura da emulsão até seu ponto de ebulição (aproximadamente, $97{ }^{\circ} \mathrm{C}$ ), mantendo-a nesta condição por determinados intervalos de tempo de forma a permitir uma perda de massa por evaporação de aproximadamente $10 \%$ a cada ciclo de aquecimento. Cada ciclo era finalizado resfriando-se a emulsão até a temperatura ambiente e obtendo-se, em seguida, seu espectro de extinção e seu tamanho médio de gota. Iniciava-se, então, o próximo ciclo de aquecimento, completando-se o ensaio quando uma perda de $90 \%$ da massa da emulsão fosse atingida. Para fins comparativos, obteve-se o tamanho médio de gota e espectros de emulsões com concentrações equivalentes àquelas obtidas ao longo da evaporação da amostra mencionada. 
2.3 Avaliação da Influência da Reposição de Água a Emulsões Submetidas a Perdas Controladas de Massa por Evaporação

Preparou-se emulsões contendo 5\% em massa do fluido de corte em estudo, totalizando $50 \mathrm{~g}$ cada uma. Um total de 10 ciclos de aquecimento foi realizado, nos quais se elevava a temperatura da amostra até seu ponto de ebulição e se iniciava o processo de resfriamento logo que $5 \%, 10 \%, 20 \%, 30 \%$, $40 \%$ ou $50 \%$ de sua massa inicial havia sido perdida, retornando-se ao final a temperatura ambiente. Em seguida, completava-se as massas perdidas adicionando-se água destilada, supondo-se que toda a perda fosse proveniente apenas da evaporação da fase contínua. Finalmente, media-se o tamanho médio de gota e o espectro de extinção, iniciando-se, em seguida, o próximo ciclo de aquecimento.

\subsection{Monitoramento da Desestabilização de} Emulsões Submetidas a Ciclos de Aquecimento em Sistema com Condensador

Acoplou-se um condensador ao erlenmeyer utilizado de forma a minimizar a perda de volume de uma emulsão de concentração mássica de $5 \%$ de fluido de corte, com uma massa inicial de 50 g. Um total de 10 ciclos foi realizado, aquecendo-se a amostra até seu ponto de ebulição e mantendo-a nesta temperatura durante $6 \mathrm{~min}$, $8,5 \mathrm{~min}, 11,5 \mathrm{~min}, 17 \mathrm{~min}, 19,5 \mathrm{~min}$ e $22 \mathrm{~min}$, intervalos estes equivalentes às perdas de massa de $5 \%, 10 \%, 20 \%, 30 \%, 40 \%$ e $50 \%$, respectivamente, como indicado no caso anterior. Em seguida, resfriava-se a emulsão até a temperatura ambiente e obtinha-se seu tamanho médio de gota e seu espectro de extinção.

\section{RESULTADOS E DISCUSSÃO}

Para todos os ensaios realizados, considerou-se que as curvas de aquecimento e resfriamento das emulsões eram aproximadamente as fornecidas na Figura 2, obtidas experimentalmente e condizentes com a obtida por Byers (2006).

Figura 2 - Curvas de aquecimento e resfriamento descritivas dos ciclos realizados com emulsões do fluido de corte em estudo

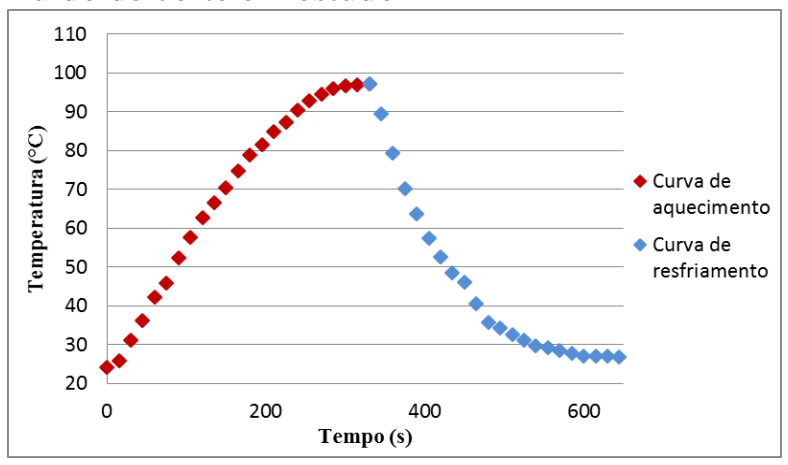

Da Figura 2 conclui-se que, para $50 \mathrm{~g}$ de emulsão, o tempo necessário para se atingir a temperatura máxima é, em média, 5 min, sendo esta o ponto de ebulição das emulsões, em torno de $97{ }^{\circ} \mathrm{C}$. Com relação ao processo de resfriamento, observa-se que são necessários, aproximadamente, $5 \mathrm{~min}$ para que uma emulsão retorne a sua temperatura inicial (admitida como sendo a temperatura ambiente).

Com relação ao monitoramento da desestabilização das emulsões, Glasse et al. (2013), verificando que os constituintes do fluido de corte em questão não absorvem luz com comprimento de onda de 400 a $900 \mathrm{~nm}$, utilizaram o intervalo de 500 a $605 \mathrm{~nm}$ para o cálculo do wavelength exponent de forma a evitar qualquer tipo de oscilação relacionada a efeitos de absorção de luz. Dessa forma, os valores de $z$ apresentados a seguir são provenientes da aplicação da Equação 1 no intervalo de comprimentos de onda mencionado, cujos resultados mostraram-se em concordância com conclusões apresentadas por outros autores (DELUHERY; RAJAGOPALAN, 2005; GLASSE et al., 2013). 


\subsection{Variação do Wavelength Exponent para uma Emulsão Submetida a Ciclos de Aquecimento em Sistema Aberto}

Valores obtidos para o wavelength exponent mostraram-se dependentes do número de ciclos de aquecimento realizados, reduzindo-se a um valor correspondente a $29 \%$ de seu valor inicial ao final do ensaio. Considerando-se este fato, afirma-se que um processo de desestabilização da emulsão está ocorrendo em consequência da coalescência das gotas da fase dispersa, como afirmado por Deluhery e Rajagopalan (2005), o que foi confirmado acompanhando-se o tamanho médio de gota ao longo dos ciclos de aquecimento, como indicado na Figura 3.

Figura 3 - Wavelength exponents e tamanhos médios de gota correspondentes para uma emulsão submetida a ciclos de aquecimento em sistema aberto

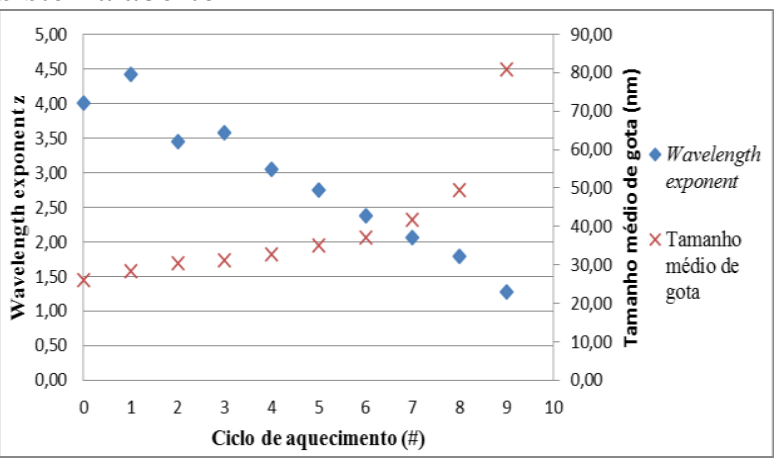

Constatou-se que até o ciclo 3 os valores do expoente $z$ oscilaram em torno do valor 4, apresentando um comportamento linear a partir de então. $O$ tamanho médio de gota, por sua vez, cresceu suavemente até o ciclo 7 , ocorrendo uma rápida elevação a partir deste até a conclusão do ensaio, como observado por Postal et al. (2015).

No entanto, deve-se considerar que a emulsão monitorada foi submetida a ciclos de aquecimento nos quais se permitiam perdas de massa para o ambiente, sendo estas de aproximadamente $10 \%$ da massa inicial em cada ciclo. Dessa forma, o processo de concentração da fase dispersa por meio da evaporação da fase contínua poderia ser uma causa adicional para o processo de desestabilização da emulsão analisada, uma vez que a quantidade disponível de emulsificantes se tornaria inadequada (HADNADEV et al., 2013) e o espaçamento entre as gotas se reduziria, aumentando-se a frequência de colisão entre estas (LIAO; LUCAS, 2010). Porém, para emulsões virgens preparadas em concentrações equivalentes àquelas obtidas ao longo dos ciclos de aquecimento, nota-se que o aumento da fração de fase dispersa não influencia isoladamente o wavelength exponent na faixa de concentrações estudada, como observado na Figura 4.

Figura 4 - Comportamento do wavelength exponent em função da concentração da fase dispersa

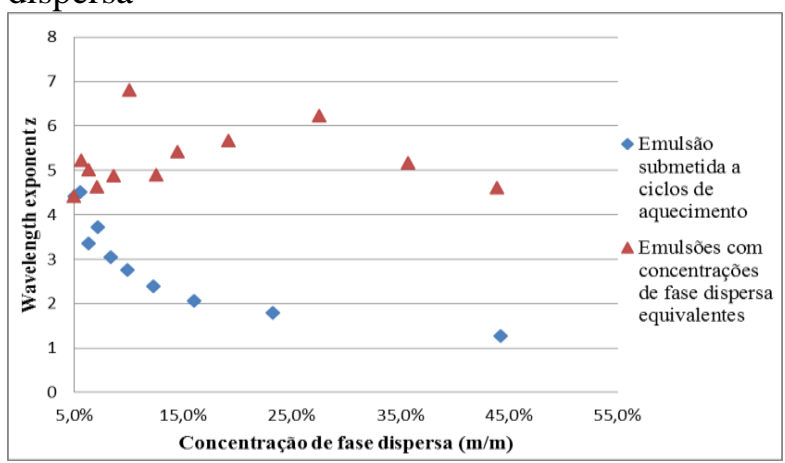

Pode-se então afirmar que outro processo atua como fator de desestabilização das emulsões, sendo importante a fração de fase dispersa apenas para concentrações acima de $45 \%$, fato este observado na Figura 5. Dessa forma, dentre os fenômenos relatados na literatura para se justificar o ocorrido, destacam-se a intensificação da hidrólise de emulsificantes e do movimento Browniano, os quais induzem o aumento da taxa de coalescência de gotas (GLASSE et al., 2014). 
Figura 5 - Tamanhos médios de gota para emulsões em diferentes concentrações

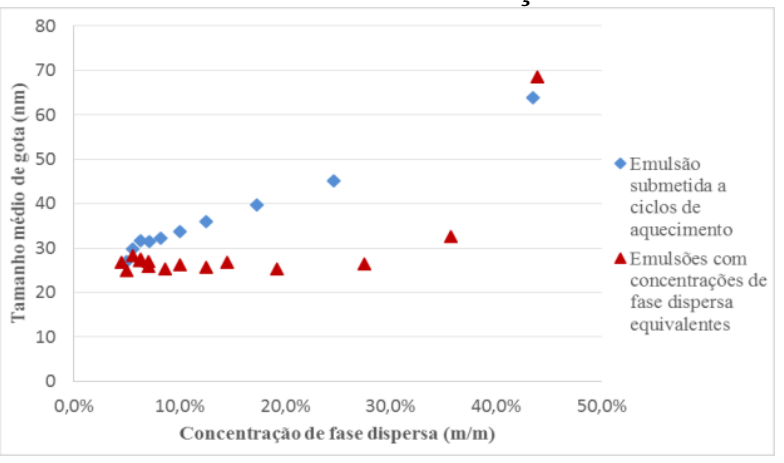

Da Figura 4 observa-se ainda que o wavelength exponent oscila em torno de um valor médio para as emulsões virgens, ou seja, não submetidas a ciclos de aquecimento e, portanto, consideradas estáveis. De acordo com Deluhery e Rajagopalan (2005), o expoente $z$ para o regime de espalhamento de luz de Rayleigh deve ser igual a 4, considerando-se este valor um indicativo da estabilidade de emulsões cujas gotas possuem dimensões compatíveis com o regime mencionado. No entanto, observa-se experimentalmente que estimativas para $\mathrm{o}$ wavelength exponent oscilam nas proximidades do valor esperado, sendo extremamente sensíveis a pequenas variações numéricas.

\subsection{Influência da Perda e Reposição de Fase Contínua na Estabilidade de uma Emulsão de Fluido de Corte}

Ao longo das etapas de evaporação e reposição da fase contínua, observou-se uma redução gradual do wavelength exponent proporcional as perdas de massa permitidas a cada ciclo, como observado na Figura 6.
Figura 6 - Wavelength exponent para emulsões submetidas a perdas de massa especificadas a cada ciclo

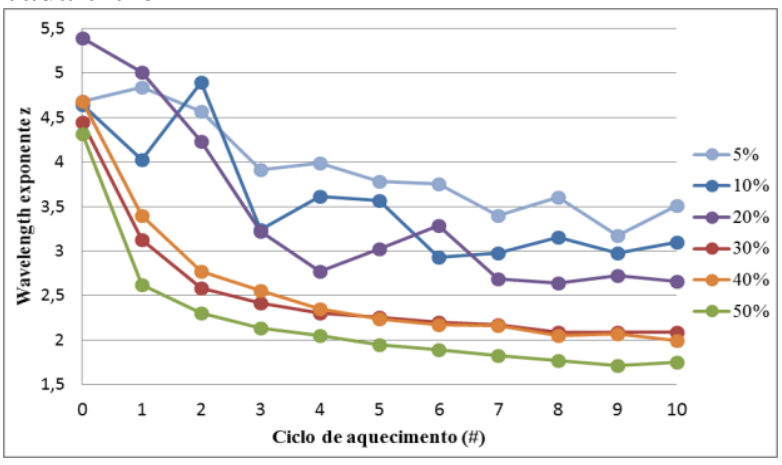

Para os casos em que as emulsões ensaiadas perdiam uma pequena quantidade de massa a cada ciclo, os valores do wavelength exponent apresentaram-se decrescentes e oscilantes quando próximos de 4. Este fato está de acordo com o mencionado anteriormente, sendo consequência de variações numéricas inerentes ao cálculo desse expoente e um indicativo de que as emulsões se encontram numa situação de proximidade à estabilidade quando comparadas às emulsões sujeitas a perdas de massa consideráveis. Para estas últimas, verificou-se quedas acentuadas de $z$ logo nos primeiros ciclos de aquecimento, com uma posterior redução suave e monotônica até a conclusão dos experimentos. Ao final, o menor coeficiente $z$ foi aquele obtido para uma emulsão na qual se perdia e se repunha $50 \%$ de sua massa inicial a cada ciclo, enquanto que o maior correspondeu a menor perda estipulada, aproximadamente $5 \%$, como pode ser observado na Figura 7.

Nota-se, portanto, que a perda e reposição de fase contínua podem atuar como fatores de aceleração da desestabilização das emulsões analisadas. Segundo Morrison e Ross (2002), a diluição de um sistema particulado pode interferir no equilíbrio dinâmico entre as moléculas de emulsificantes adsorvidas na superfície das partículas e livres na fase contínua, sendo possível de ser interpretada como mais um fator impactante 
para a estabilidade de uma emulsão. Uma vez que a reposição da fase contínua se dava pela adição de água destilada, ou seja, livre de qualquer tipo de emulsificante, não se pode desconsiderar esta hipótese ao longo da interpretação dos valores de $z$ obtidos.

Figura 7 - Valores finais do wavelength exponent e do tamanho médio de gota para os ensaios realizados com perdas de massa específicas

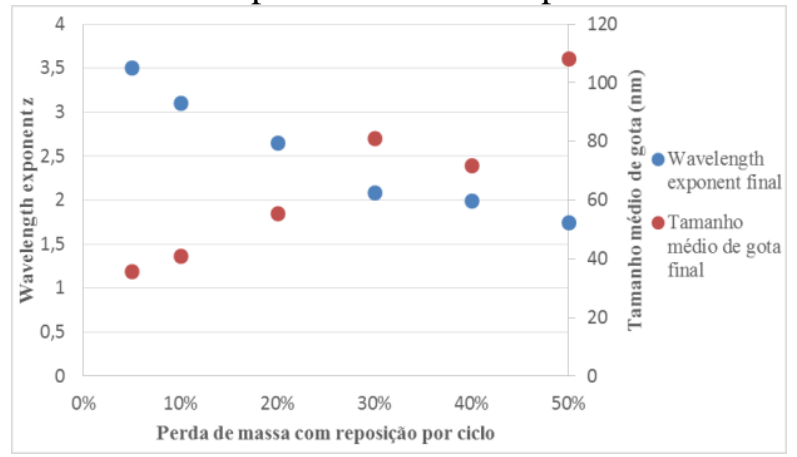

3.3 Variação do Wavelength Exponent para uma Emulsão Submetida a Ciclos de Aquecimento em Sistema com Condensador Acoplado

O acoplamento de um condensador ao erlenmeyer utilizado para aquecer as emulsões possibilitou a minimização da perda de massa por evaporação para aproximadamente $2 \%$ de sua massa inicial ao final dos ensaios. Dessa forma, eliminou-se os processos de concentração e diluição como fatores que poderiam contribuir para a desestabilização das emulsões, estando estas sujeitas apenas a ação do aquecimento e resfriamento ao longo dos ciclos. Na Figura 8 pode-se observar os valores do wavelength exponent obtidos para ciclos com tempos de aquecimento próximos aos necessários para se obter as perdas de massa por evaporação indicadas anteriormente.
Figura 8 - Wavelength exponent para emulsões submetidas a tempos de aquecimento equivalentes as perdas de massa indicadas na Figura 6

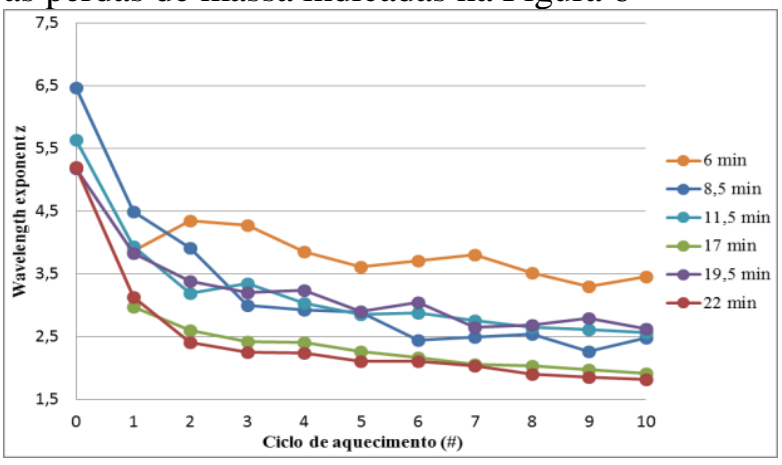

Nota-se novamente que o valor do wavelength exponent se reduz ao longo dos ciclos, apresentando certa oscilação quando próximo do valor 4 , como observado para tempos de aquecimento menores, opondo-se a característica monotônica observada para tempos mais longos. Com exceção da curva obtida para aquecimentos de 19,5 min, muito provavelmente consequente de erro experimental, as demais se comportaram como o esperado, apresentando reduções nos valores do wavelength exponent proporcionais aos intervalos de aquecimento. Os valores finais deste expoente e dos tamanhos médios de gota estão resumidos na Figura 9.

Figura 9 - Valores finais do wavelength exponent e do tamanho médio de gota para os ensaios realizados com tempos de aquecimento específicos

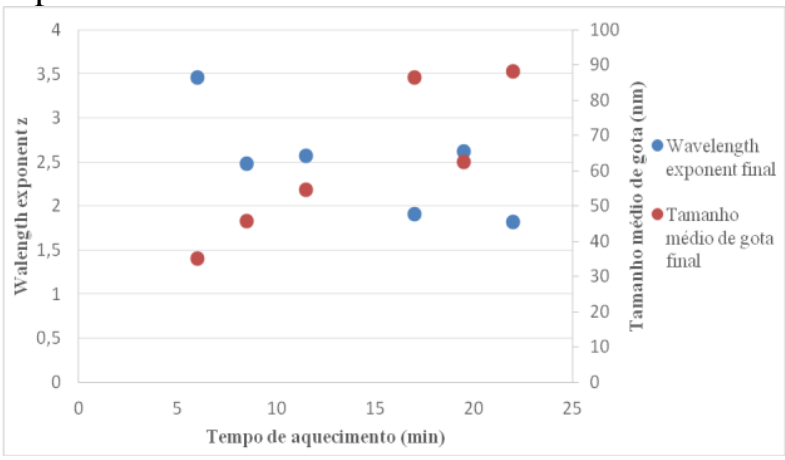

Comparando-se os casos equivalentes em que as emulsões eram submetidas a tempos de aquecimento ou perdas de massa 
por ciclo determinados, observa-se uma considerável proximidade entre os valores de wavelength exponent obtidos, como indicado nas Figuras 10-15.

Figura 10 - Comparação entre sistemas equivalentes: tempo de aquecimento de 6 min e perda de massa por ciclo de $5 \%$

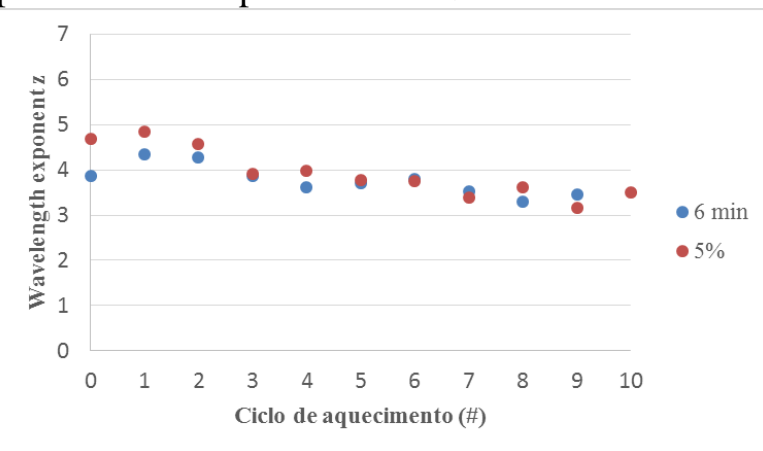

Figura 11 - Comparação entre sistemas equivalentes: tempo de aquecimento de 8,5 min e perda de massa por ciclo de $10 \%$

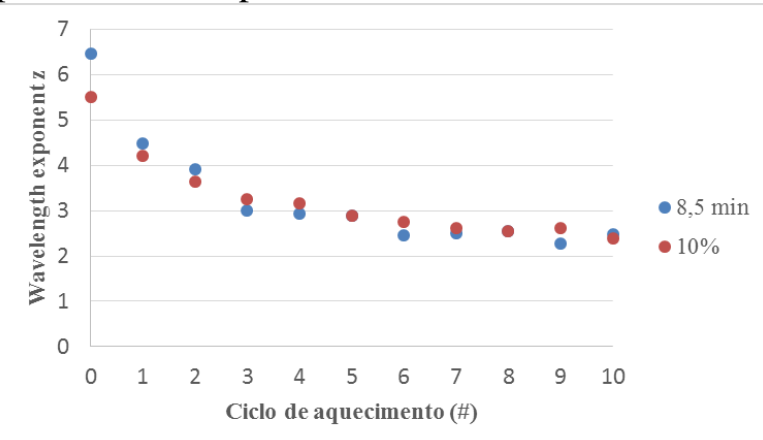

Figura 12 - Comparação entre sistemas equivalentes: tempo de aquecimento de $11,5 \mathrm{~min}$ e perda de massa por ciclo de $20 \%$

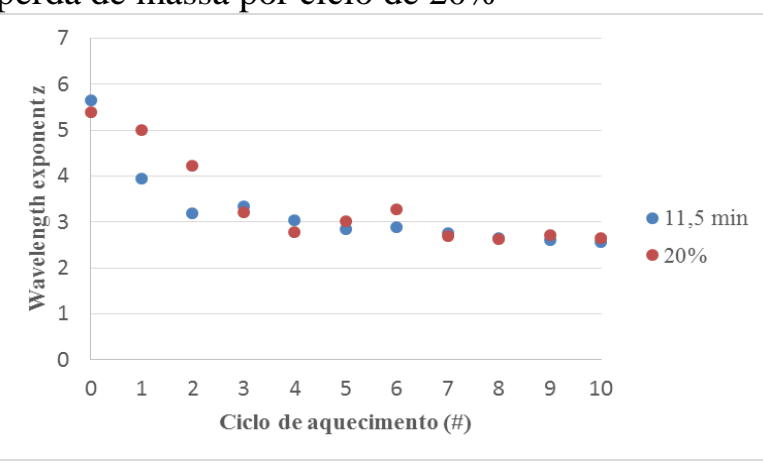

Figura 13 - Comparação entre sistemas equivalentes: tempo de aquecimento de $17 \mathrm{~min}$ e perda de massa por ciclo de $30 \%$

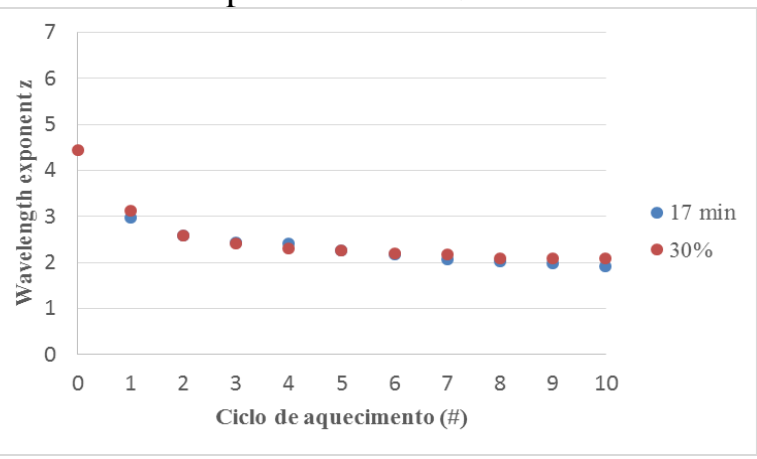

Figura 14 - Comparação entre sistemas equivalentes: tempo de aquecimento de 19,5 min e perda de massa por ciclo de $40 \%$

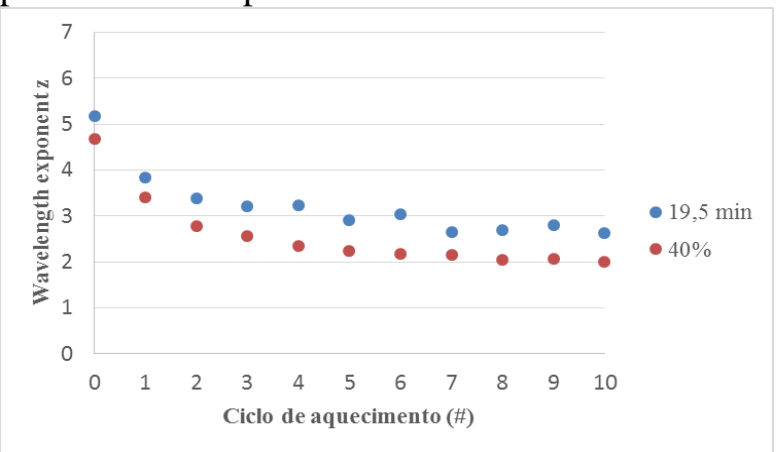

Figura 15 - Comparação entre sistemas equivalentes: tempo de aquecimento de $22 \mathrm{~min}$ e perda de massa por ciclo de $50 \%$

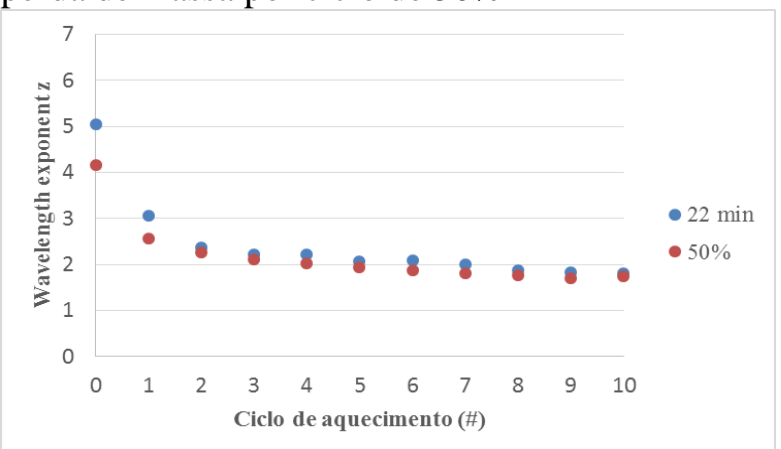

Nota-se que há uma concordância entre os valores obtidos para sistemas nos quais os tempos de aquecimento eram semelhantes, com exceção do caso indicado pela Figura 14, cujo distanciamento da tendência observada pode ser justificado pela observação de resultados contraditórios para tempos de 
aquecimento de 19,5 min a cada ciclo, provavelmente consequentes de erro experimental, como mencionado anteriormente.

A semelhança entre os valores do wavelength exponent minimiza a possibilidade de que perdas de massa por evaporação e reposição de fase contínua, a qual provocava a diluição das emulsões ao final de cada ciclo, sejam fatores de grande impacto na estabilidade das mesmas. Portanto, pode-se afirmar que de todos os fatores mencionados, o tempo de aquecimento é o mais relevante para a desestabilização das emulsões estudadas.

\section{CONCLUSÕES}

A submissão de emulsões de um fluido de corte a ciclos de aquecimento mostrou-se como sendo um fator acelerador da desestabilização desses sistemas, processo esse possível de ser monitorado via espectroscopia UV-Vis pelo cálculo do wavelength exponent dos espectros de extinção ao longo do tempo.

Para sistemas abertos nos quais a fração

de fase dispersa na emulsão era continuamente aumentada devido à perda de fase contínua por evaporação, notou-se a redução do coeficiente $z$ ao longo dos ciclos, o que foi acompanhado pelo aumento do tamanho médio de gota. Verificou-se também que o aumento da fração de fase dispersa não representa um fator de contribuição para o processo de desestabilização desses sistemas na faixa de concentrações analisada, podendo este fenômeno ser atribuído a intensificação da hidrólise de emulsificantes e do movimento Browniano, os quais induzem o aumento da taxa de coalescência de gotas.

Perdas de massa controladas de fase contínua e sua reposição ao final de cada ciclo resultaram na redução do wavelength exponent de modo gradual, observando-se valores menores deste expoente para as maiores perdas estipuladas. Observou-se também que o wavelength exponent apresenta comportamento oscilante em alguns casos, resultante de pequenas variações numéricas inerentes de seu cálculo, o que se observa com mais intensidade nas proximidades do valor 4.

Acoplando-se um condensador ao sistema, foi possível minimizar as perdas de massa por evaporação a cada ciclo, sujeitando as emulsões exclusivamente ao efeito da temperatura. Dessa forma, notou-se a redução gradual do coeficiente $z$ proporcionalmente ao tempo de aquecimento das emulsões. Comparando-se com os casos em que se especificou as perdas de massa, concluiu-se que a diluição das emulsões ao final de cada ciclo não representou um fator impactante para a desestabilização das emulsões, sendo este fenômeno induzido principalmente pelo efeito da exposição a elevadas temperaturas.

\section{NOMENCLATURA}

$\begin{array}{ll}\tau & \text { Turbidez } \\ \lambda & \text { Comprimento de onda da luz, } n m \\ z & \text { Wavelength exponent }\end{array}$

\section{REFERENCIAS}

ASSENHAIMER, C.; MACHADO, J. M.; GLASSE, B; FRITSCHING, U; GUARDANI, R. Use of a Spectroscopic Sensor to Monitor Droplet Size Distribution in Emulsions Using Neural Networks. The Canadian Journal of Chemical Engineering, v. 92, n. 2, p. 318-323, 2013.

BAUMEISTER, T.; AVALLONE, E. A.; BAUMEISTER III, T. Marks' Standard Handbook for Mechanical Engineers. 8. ed. McGraw Hill Book Co, 1978. 1850 p.

BYERS, J. P. Metalworking Fluids. 2. ed. Boca Raton: CRC Press, 2006. 450 p. 
DELUHERY, J.; RAJAGOPALAN, N. A turbidimetric method for the rapid evaluation of MWF emulsion stability. Colloids and Surfaces A: Physicochemical and Engineering Aspects, v. 256, n. 2-3, p. 145149, 2005.

GLASSE， B.; ASSENHAIMER, C.; GUARDANI, R.; FRITSCHING, U. Analysis of the Stability of Metal Working Fluid Emulsions by Turbidity Spectra. Chemical Engineering \& Technology, v. 36, n. 7, p. 18, 2013.

GLASSE, B.; ASSENHAIMER, C.; GUARDANI, R.; FRITSCHING, U. Turbidimetry for the Stability Evaluation of Emulsions Used in Machining Industry. The Canadian Journal of Chemical Engineering, v. 92, n. 2, p. 324-329, 2014.

HADNADEV, T. D.; DOKIC, P.; KRSTONOSIC, V.; HADNADEV, $\mathrm{M}$. Influence of oil phase concentration on droplet size distribution and stability of oil-inwater emulsions. European Journal of Lipid Science and Technology, v. 115, n. 3, p. 313321, 2013.

JOKISCH GmbH. Jokisch Technisches Merkblatt - Jokisch Kompakt YV neu, 2014. Disponível em: <http://jokischfluids.de/uploads/Kompakt_YV_neu_01.pdf > Acesso em: 6 jun. 2015.

LIAO, Y.; LUCAS, D. A literature review on mechanisms and models for the coalescence process of fluid particles. Chemical Engineering Science, v. 65, n. 10, p. 28512864, 2010.

MORRISON, I. D.; ROSS, S. Colloidal Dispersions: Suspensions, Emulsions and Foams. 1. ed. Nova York: John Wiley and Sons, Inc., 2002, 616 p.
OLIVEIRA, J. F. DE; ALVES, S. M. Adequação ambiental dos processos de usinagem utilizando Produção mais Limpa como estratégia de gestão ambiental. Produção, v. 17, n. 1, p. 129-138, 2007.

POSTAL, V.; CORREIA, J. C.; ASSENHEIMER, C.; GUARDANI, R. Estudo da degradação térmica de emulsões de fluidos de corte, p. 11993-12000 . In: Anais do XX Congresso Brasileiro de Engenharia Química - COBEQ 2014 [= Blucher Chemical Engineering Proceedings, v.1, n.2]. São Paulo: Blucher, 2015.

REDDY, S. R.; FOGLER, H. S. Emulsion Stability: Determination from Turbidity. Journal of Colloid and Interface Science, v. 79, n. 1, p. 101-104, 1981.

\section{AGRADECIMENTOS}

Os autores gostariam de agradecer a Jokisch GmbH pela doação das amostras de fluido de corte e à CAPES e CNPq pelo apoio a esta pesquisa. 